\title{
Corynebacterium bovis
}

National Cancer Institute

\section{Source}

National Cancer Institute. Corynebacterium bovis. NCI Thesaurus. Code C86311.

A species of aerobic or facultatively anaerobic, Gram-positive, coryneform, pleomorphic bacilli assigned to the phylum Actinobacteria. This species is nonmotile, non-spore forming, catalase positive, oxidase positive, slow growing, urease positive, and lipophilic. C. bovis is commensal to the bovine udder, but can be pathogenic in bovine and other species, including humans. 